\title{
Neu entdeckte Fragmente der ältesten bekannten ,Lucidarius'-Handschrift
}

\author{
Lukas Wolfinger Mona Alina Kirsch
}

$\mathrm{D}$ ie Entstehungs- und Textgeschichte des deutschen,Lucidarius' gibt der Forschung nach wie vor Rätsel auf. ${ }^{.}$Neue Impulse erfuhr die Diskussion vor allem durch die Arbeiten von Georg Steer und Dagmar Gottschall, die alte Gewissheiten bezüglich des Entstehungskontextes und der Überlieferung in Frage stellten. ${ }^{2}$ Doch so umstritten die Ursprünge dieses bedeutenden mittelalterlichen Werkes auch sind, so unbestritten stellt die ,Fragmente-Handschrift ${ }^{\prime} 2^{\circ}$ Cod. Ms. theol. IoIn Cim. der Staats- und Universitätsbibliothek Göttingen den ältesten bekannten Textzeugen dar. ${ }^{3}$ Umso bedauerlicher musste es erscheinen, dass sowohl zur Überlieferungsgeschichte als auch zur Provenienz dieser Zimelie aus der Zeit um I200, deren Blätter zum einen Teil in Heidelberg, zum anderen in Göttingen gefunden wurden, so gut wie nichts bekannt ist. Im Rahmen des DFG-geförderten Projekts zur Neukatalogisierung des mittelalterlichen Handschriftenbestandes der Niedersächsischen Staatsund Universitätsbibliothek Göttingen, das 2017 in der Abteilung, Spezialsammlungen und Bestandserhaltung seine Arbeit aufnahm, ${ }^{4}$ sind nun jedoch neue Ergebnisse und Funde möglich geworden.

Von den zuerst entdeckten Heidelberger Stücken wusste man nur, dass der Archivar und Historiker Franz Joseph Mone (1796-I87I) sie vor I834 „von einem alten Buchdeckel in Heidelberg" "` abgelöst hatte, aber nicht aus welchem Buch. Ihre Herkunft lag demnach im Dunklen. Für die in Göttingen entdeckten Fragmente war immerhin bekannt, dass sie aus einer Inkunabel stammen, die aus der Bibliothek der Grafen von Starhemberg an die Georg-August-Universität gelangte. ${ }^{6}$ Diese 1477 in Basel gedruckte ,Lectura super quinque libros Decretalium' des Nicolaus de Tudeschis trägt heute die Göttinger Signatur $2^{\circ}$ Jus canon. 35/42 (Kind Nr. 1374).7 Ihr Einband ist u. a. mit einem Blindstempel geschmückt, der eine Buchstabenkombination zeigt, die schon Karl Schorbach in einer Fußnote seiner I894 erschienenen ,Studien über das deutsche Volksbuch Lucidarius' erwähnte. Er las sie als „iod* esth“ und interpretierte sie als Abkürzung für den Namen des ehemaligen Besitzers der Inkunabel bzw. desjenigen, der den Einband in Auftrag gegeben hatte: Jodocus Esth. ${ }^{8}$ Noch Helgard Ulmschneider folgt Schorbach in ihrer Monographie zur mittelalterlichen Überlieferungsgeschichte des ,Lucidarius' in dieser Lesart. ${ }^{9}$ Betrachtet man den betreffenden Einbandstempel genauer, ist jedoch zu erkennen, dass die genannte Buchstabenkombination zwar durchaus als „Esth“ gelesen werden kann, ebenso gut aber auch als „Esch“ (Abb. I). Die bislang als „st" interpretierten Buchstaben wären dann als „sc“-Ligatur zu deuten. In der Einbanddatenbank findet sich ein Jodocus Esch zwar ebenso wenig wie ein Jodocus Esth, doch wird man anderwärtig fündig: Ernst Kyriss führt unter den von ihm gesammelten Belegen zu Einbandwerkstätten und -stempeln auch einen Buchbinder namens "Jodocus Esch“, von dem er vermutet, dass er in Basel tätig war. ${ }^{\circ}$

Nachdem die Einbandwerkstatt der Göttinger Inkunabel nachgewiesen war, erschien es geboten, den übrigen bislang bekannten Erzeugnissen derselben nachzuspüren. Allerdings nennt Ernst Kyriss in seinen diesbezüglichen Publikationen nur zwei entsprechende Stücke: Dabei handelt 
es sich um eine $1472 / 73$ in Basel gedruckte Bibel in zwei Teilen, die unter der Signatur Q 300-III Folio INC an der Universitätsbibliothek Heidelberg aufbewahrt wird, ${ }^{\text {II }}$ also ausgerechnet an jenem Ort, an dem Franz Joseph Mone einst die ersten beiden Fragmente des ,Lucidarius' entdeckt hatte. Damit lag die Vermutung sehr nahe, eine dieser beiden Inkunabeln könne den bislang nicht nachgewiesenen Trägerband der ,Lucidarius'Blätter gebildet haben. Der Blick in den Heidelberger Inkunabelkatalog verstärkte diese Vermutung, denn hier werden bei diesem zweiteiligen Bibeldruck nicht nur „Fragmente verschiedener Pergamenthandschriften " erwähnt, sondern auch ein Abklatsch auf dem Vorderspiegel des ersten Bandes. ${ }^{12}$ Da Mone die Fragmente laut eigenen Aussagen einem Einband entnommen hatte, erschien es gut möglich, dass dieser Abklatsch von einem der beiden Stücke herrührte. Da die Suche nach weiteren Werken aus der Werkstatt ,Jodocus Esch' offenbar ausgerechnet zu jenen Bänden führte, die schon Franz Joseph Mone in Händen gehalten hatte, sank zugleich freilich die Hoffnung, darin noch weitere Fragmente der ,Lucidarius'-Handschrift zu entdecken. Erst der Blick in die originalen (Ein-)Bände konnte zeigen, ob die Hoffnungen auf weitere Fragmente begründet und die Vermutungen zum Trägerband der Heidelberger Stücke zutreffend waren.

Tatsächlich bestätigte ein genauer Blick in den Band, der den Abklatsch enthält, unsere Vermutungen gleich in zweifacher Hinsicht: Zum einen stammt der Abklatsch, wie die Spiegelung desselben erweist, tatsächlich von den Fragmenten, die über Franz Joseph Mone und Karl Schorbach nach Göttingen gelangten. Zweifelsfrei erkennbar ist das bei der rechten unteren Ecke von Fragment M/S 2r, die auf der Innenseite des Vorderdeckels ihre klare Entsprechung findet (Abb. $2 a$ und $2 b$ ). Zum anderen erwies sich auch die Annahme als zutreffend, dass Mone den Band bereits gut durchforstet und keine weiteren Fragmente in dieser Handschrift belassen bzw. gefunden hatte. Ungleich mehr Erfolg war der
Suche jedoch im zweiten Band der Inkunabel beschieden - ihn hat Mone offenkundig nicht untersucht: Wie schon dem Inkunabelkatalog zu entnehmen war, weist dieser Band Papier- und Pergamentfragmente unterschiedlicher Herkunft auf. Bei mehreren Stücken fiel sofort die Ähnlichkeit mit der ,Lucidarius'-Handschrift auf und unschwer ließ sich erkennen, dass diese einen deutschen Text enthalten. Schnell sprangen auch die charakteristischen Abkürzungen [D]o. s. d.J. und $[D]$ o. s. d. M. ins Auge, die für die Worte Do sprach der Junger und Do sprach der Meister stehen und im Text des Werkes so häufig wiederkehren, wobei die eingeplanten farbigen Lombarden - so wie üblich im Göttinger Textzeugen - nicht ausgeführt sind. Damit war klar, dass die entsprechenden Fragmente in diesem Band der Werkstatt ,Jodocus Esch' tatsächlich weitere Überreste der ältesten deutschen ,Lucidarius'-Handschrift darstellen.

Es handelt sich dabei erstens um drei größere Stücke, die im vorderen wie hinteren Einbanddeckel eingeklebt und dazu gedacht sind, die Verbindung des Buchblocks mit den Deckeln zu stärken. Zwei von ihnen kleben im Vorderdeckel und reichen hinter dem Buchblock hindurch zu den Blättern 20 und 2I, zwischen denen sie mit einem schmalen Streifen hochkommen. Das obere Fragment misst ca. $180 \times 80-90 \mathrm{~mm}$, das untere etwa $190 \times 55-65 \mathrm{~mm}$. Der Text des Letzteren konnte bereits identifiziert werden. ${ }^{13}$ Das Stück im hinteren Deckel ist etwas größer, nämlich ca. $230 \times 78-88 \mathrm{~mm}$ und reicht zurück bis zwischen die Blätter 200 und 201 der Inkunabel. Das Pergamentblatt, das oberhalb davon in den Deckel geklebt ist, gehört hingegen deutlich erkennbar zu einem anderen Schriftstück (Abb. 3). Im Bereich der Einbanddeckel sind all diese Fragmente zum Großteil von den Spiegelblättern überklebt, unter denen sich - soweit erkennbar - wohl keine weiteren Stücke mehr verbergen. $\mathrm{Zu}$ diesen drei Blättern kommt zweitens noch eine größere Zahl von schmalen Pergamentstreifen hinzu, die zur Falzverstärkung in der Mitte der Lagen ver- 
wendet wurden (Abb. 4). Wenigstens in der ersten Hälfte des Bandes stammen diese wohl vorrangig oder zur Gänze aus der ,Lucidarius'-Handschrift.

Zwar werden erst weitere Untersuchungen erweisen können, ob und inwieweit die neu entdeckten Fragmente ein neues Licht auf die Textfassung und Geschichte der Handschrift, aus der sie stammen, werfen können oder gar auf die allgemeine Text- und Überlieferungsgeschichte des deutschen ,Lucidarius'. In jedem Fall aber hat dieser Heidelberger Neufund die aus der Einband- und Provenienzanalyse gewonnenen Überlegungen zum ursprünglichen Trägerband der von Mone entdeckten ,Lucidarius'-Fragmente vollauf bestätigt. Die Frage, wo bzw. in welchem Buch Franz Joseph Mone die zuerst entdeckten Teile der Göttinger ,Lucidarius'-Handschrift gefunden hat, kann nun als geklärt gelten. Da- durch ergeben sich zugleich weitere Möglichkeiten, den Kontext, in dem die ,Lucidarius'-Handschrift makuliert wurde, genauer zu konturieren. Ein ausführlicher Beitrag zu den buchhistorischen und sozialen ,Verbindungen', in denen die neu aufgefundenen Fragmente zu sehen sind, ist deshalb bereits in Vorbereitung. Und schließlich sind durch die neuen Ergebnisse zur Einbandwerkstatt, in der die Fragmente zweitverwendet wurden, die Chancen merklich gestiegen, noch weitere Stücke der ältesten bekannten ,Lucidarius-Handschrift ausfindig zu machen. Somit bleibt, wie schon Karl Schorbach vor rund I30 Jahren erklärte, zu hoffen, dass „ein glücklicher Zufall auch noch die übrigen zerschnittenen Blätter des Manuskriptes zu Tage fördern kann" ${ }^{{ }^{14}}$ - und wenn schon nicht der Zufall, dann eben Langzeit-Erschließungsprojekte.

\section{Kontakt}

Lukas Wolfinger · Mona Alina Kirsch

Niedersächsische Staats- und Universitätsbibliothek Göttingen· Papendiek I4 · 37073 Göttingen

E-Mail:wolfinger@sub.uni-goettingen.de·kirsch@sub.uni-goettingen.de

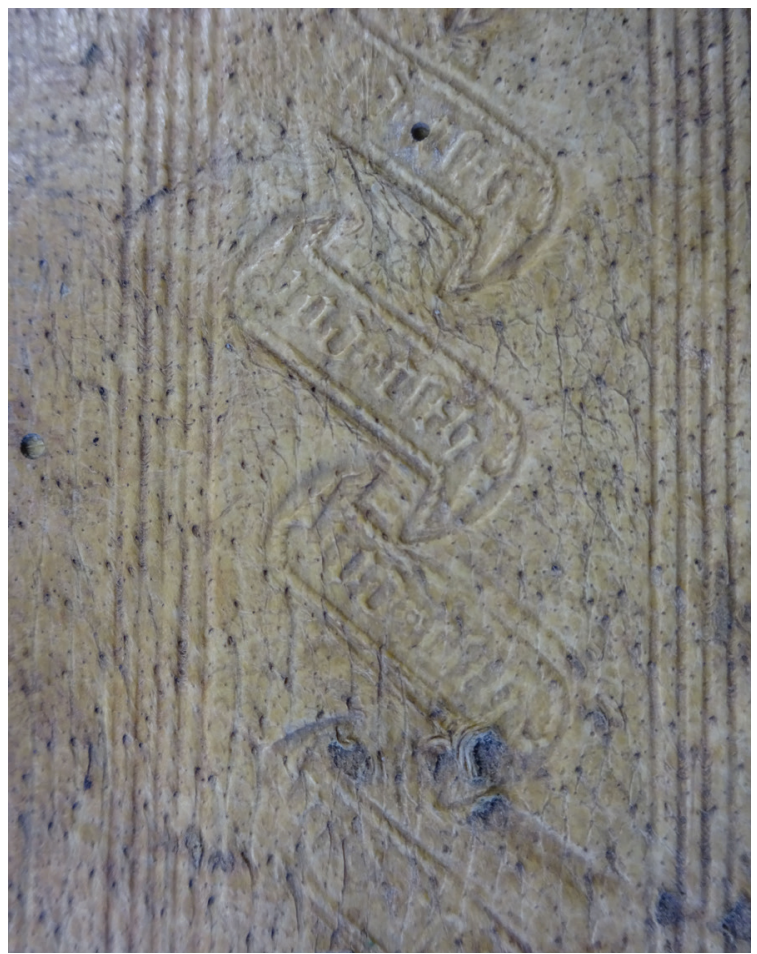

Abb. I: Stempel „iod. esch“ auf dem Einband von Göttingen, Staatsund Universitätsbibliothek, $2^{\circ}$ Jus canon. $35 / 42$ (Kind Nr. 1374) 


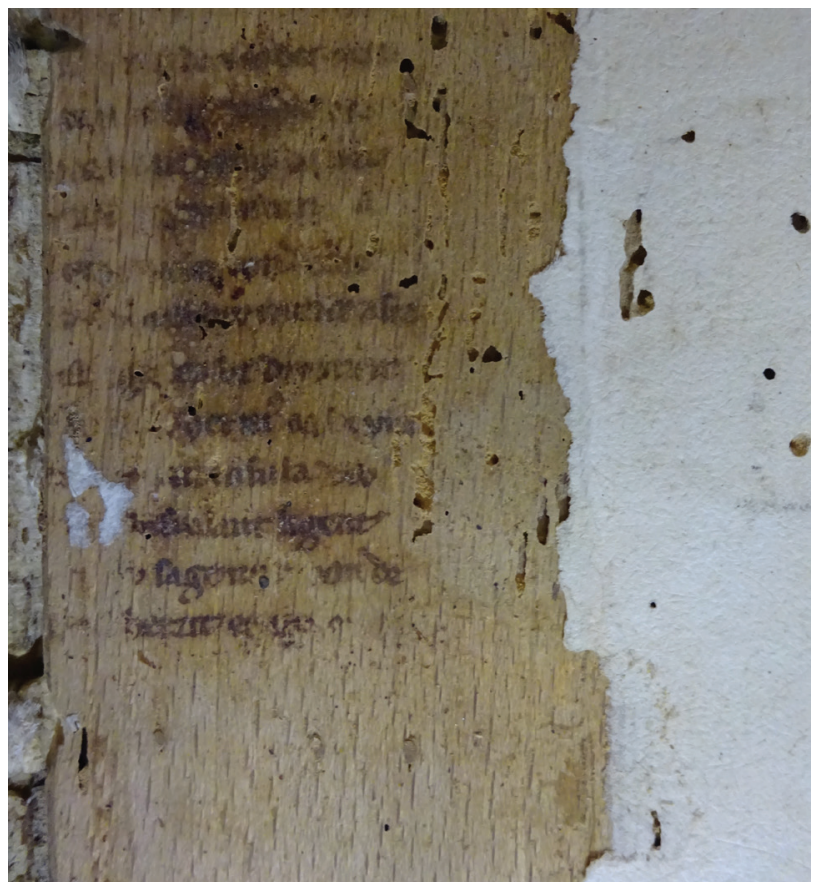

Abb. 2a: Abklatsch-Spiegelung zum vorderen Deckel von Heidelberg, Universitätsbibliothek, Q 300-I-II Folio INC, Bd. I

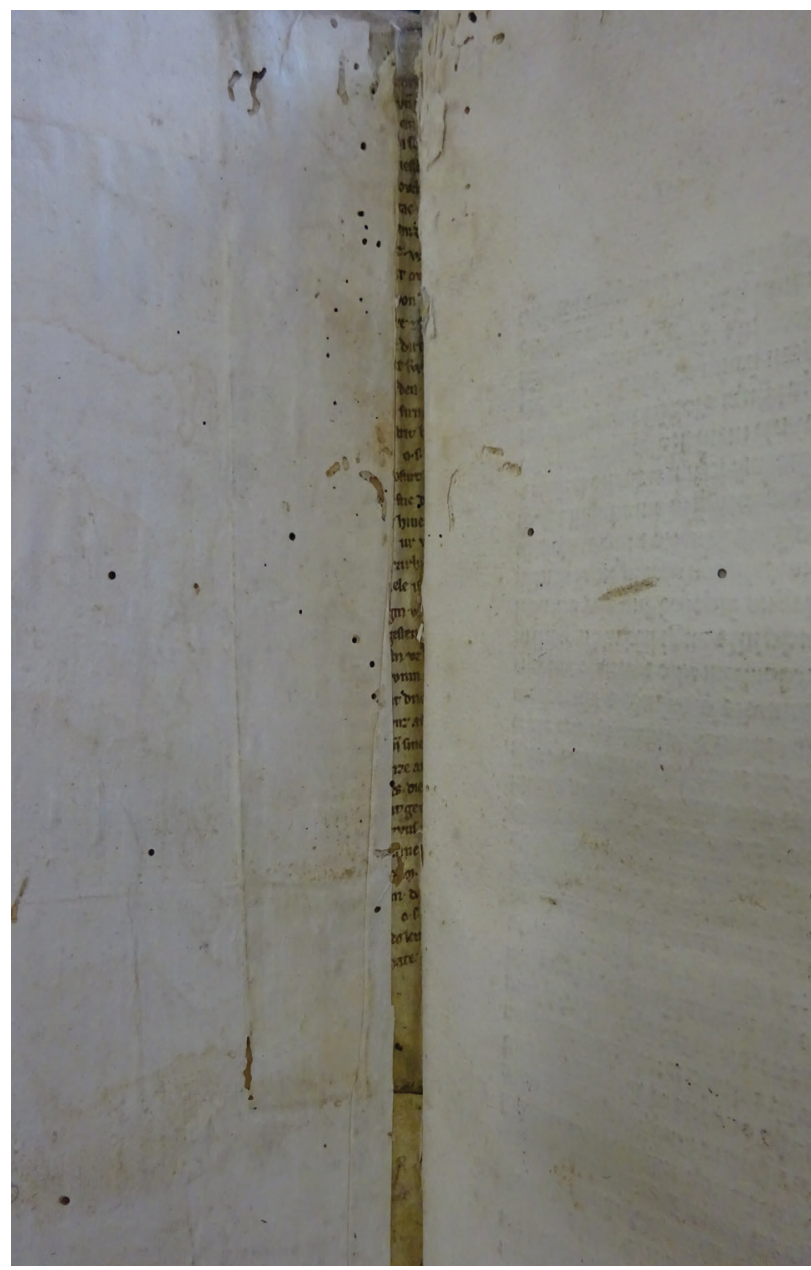

Abb. 3: Pergament-Fragment im hinteren Einbanddeckel von Heidelberg, Universitätsbibliothek, Q 300-I-II Folio INC, Bd. 2

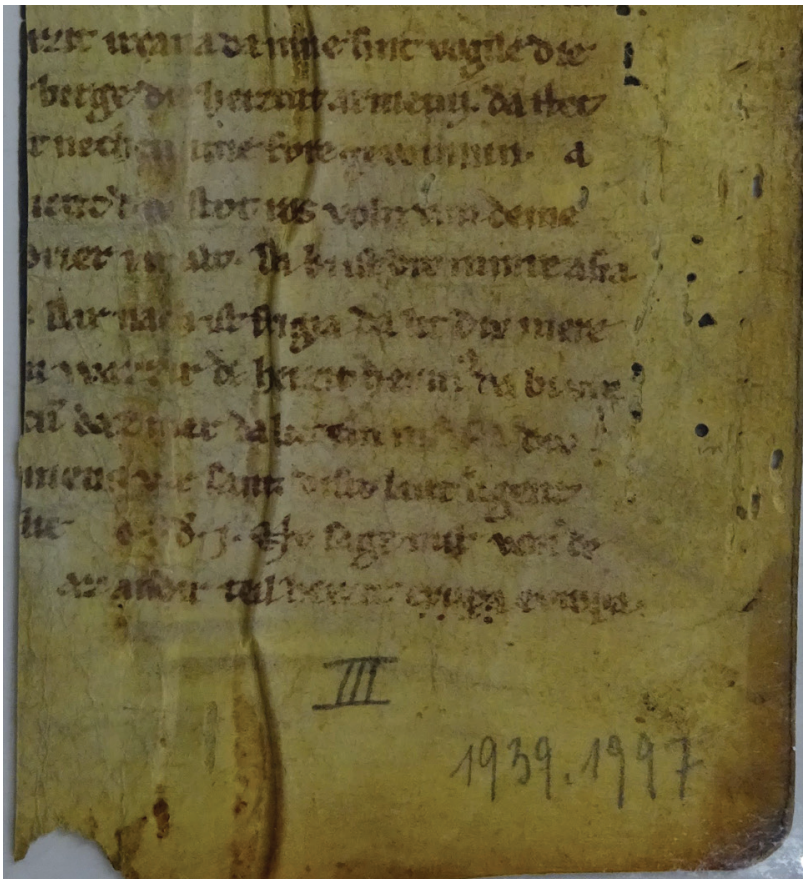

Abb. 2b: Original-Fragment M/S 2r aus Göttingen, Staats- und Universitätsbibliothek, $2^{\circ}$ Cod. Ms. theol. IoIn Cim.

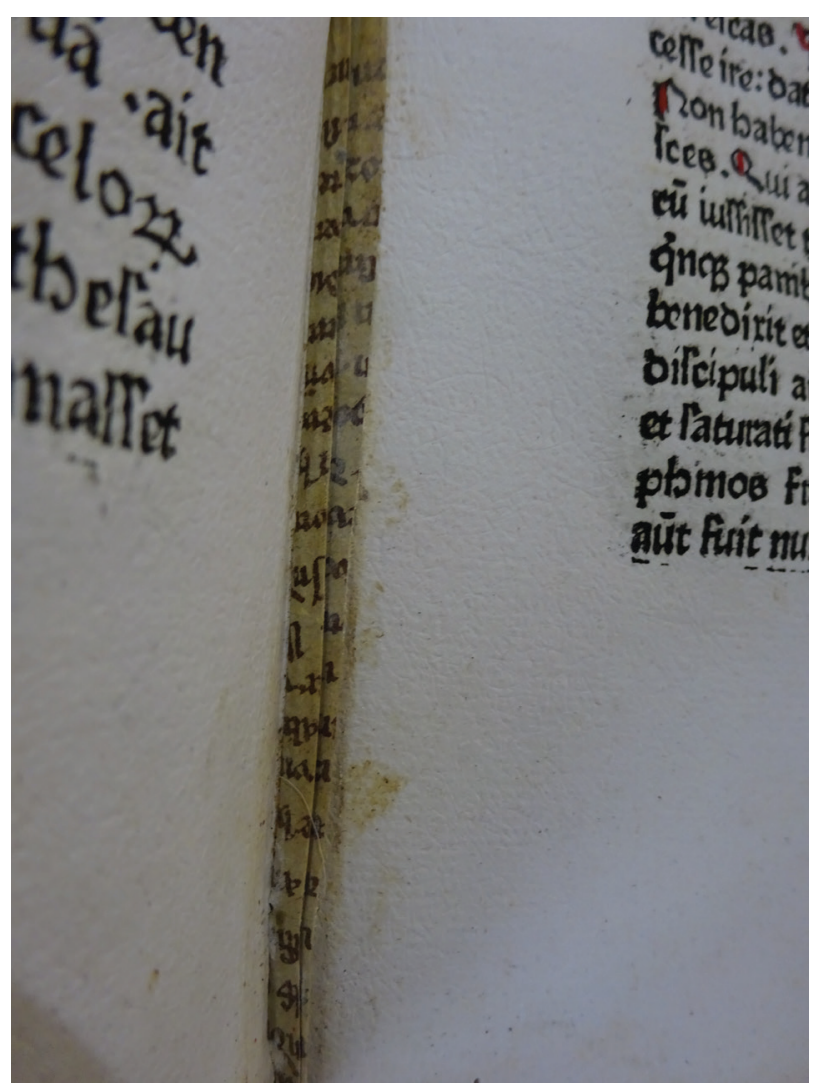

Abb. 4: Pergamentstreifen zur Falzverstärkung in Heidelberg, Universitätsbibliothek, Q $300-\mathrm{I}$-II Folio INC, Bd. 2 


\section{Anmerkungen}

I Einen kurzen Überblick zum deutschen ,Lucidarius‘ bietet Sabina Foidl, Lucidarius, in: Deutsches Literatur-Lexikon. Das Mittelalter, hg. von Wolfgang Achnitz, Bd. I: Das geistliche Schrifttum von den Anfängen bis zum Beginn des I4. Jahrhunderts, Berlin/New York 20II, Sp. 558-56I; zur grundlegenden älteren Literatur vgl. insbesondere: Karl Schorbach, Studien über das deutsche Volksbuch Lucidarius und seine Bearbeitungen in fremden Sprachen (Quellen und Forschungen zur Sprach- und Culturgeschichte der germanischen Völker 74), Straßburg ı894, sowie Felix Heidlauf, Das mittelhochdeutsche Volksbuch Lucidarius, Diss. Berlin i9ı; zum Text und seiner Überlieferung nun v. a.: Dagmar Gottschall und Georg Steer (Hg.), Der deutsche ,Lucidarius‘, Bd. I: Kritischer Text nach den Handschriften (Texte und Textgeschichte 35), Tübingen I994. Marlies Hamm, Der deutsche ,Lucidarius‘, Bd. 3: Kommentar (Texte und Textgeschichte 37), Tübingen 2002; Helgard Ulmschneider, Der deutsche ,Lucidarius', Bd. 4: Die mittelalterliche Überlieferungsgeschichte (Texte und Textgeschichte 38), Berlin/New York 2011 .

2 Vgl. Gottschall/Steer [Anm. I]; zur Frage der Entstehungsgeschichte vgl. insbesondere: Georg Steer, Der deutsche Lucidarius. Ein Auftragswerk Heinrichs des Löwen?, in: Deutsche Vierteljahrsschrift für Literaturwissenschaft und Geistesgeschichte 64 (I990), S. I-25; Helgard Ulmschneider, Wistůmes vil, vremde mere oder zum zit vertriben? Zur Überlieferung der Prologe des deutschen ,Lucidarius' in den mittelalterlichen Handschriften, in: ZfdA I38 (2009), S. I4I-I65; Christa Bertelsmeier-Kierst, Fern von Braunschweig und fern von Herzogen Heinriche? Zum A-Prolog des „Lucidarius“, in: Zeitschrift für deutsche Philologie I22 (2003), S. 20-47; Werner Schröder, Textkritisch oder überlieferungskritisch: zur Edition des deutschen ,Lucidarius', in: Critica selecta. Zu neuen Ausgaben mittelhochdeutscher und frühneuhochdeutscher Texte, hg. von Werner Schröder u.a. (Spolia Berolinensia I4), Hildesheim u. a. I999, S. 75-III.

3 Dazu sowie insgesamt zur Göttinger Fragmente-Handschrift des deutschen ,Lucidarius' siehe Schorbach [Anm. I], S. I9f. und S. 269-272; Heidlauf [Anm. I], S. 4, Nr. I und S. 34-39; Gottschall/Steer [Anm. I], S. I2*, Nr. 20 und S. $4 \mathrm{I}^{*}-44^{*}$; Ulmschneider [Anm. I], S. III-II4; Franz Joseph Mone, Altteutsche Weltlehre des Lucidarius, in: Anzeiger für Kunde des deutschen Mittelalters 3 (I834), Sp. 3II-320; Wilhelm Meyer, Die Handschriften in Göttingen, Bd. 3: Universitäts-Bibliothek. Nachlässe von Gelehrten - Orientalische Handschriften - Handschriften im Besitz von Instituten und Behörden - Register (Verzeichniss der Handschriften im Preußischen Staate I,3), Berlin ı894, S. 545; Marlies Dittrich, Zur ältesten Überlieferung des deutschen Lucidarius, in: ZfdA 77 (1940), S. 218-255; Helgard Ulmschneider, Von der ordenunge dirre welte. Zur ältesten Weltkarte in einem deutschsprachigen Text, der mappa mundi im ,Lucidarius', in: Vom vielfachen Schriftsinn im Mittelalter. Festschrift für Dietrich Schmidtke, hg. von Freimut Löser und Ralf G. Päsler (Schriften zur Mediävistik 4), Hamburg 2005, S. 579-605, hier S. $580 f .$, S. 585 und S. 603 (Abb. 4); Katalog der deutschsprachigen illustrierten Handschriften des Mittelalters, begonnen von Hella Frühmorgen-Voss und Norbert H. Ott, hg. von Kristina Freienhagen-Baumgardt, Pia Rudolph und Nicola Zotz, Bd. 8,5 (Lucidarius - Johann von Soest, ,Margreth von Limburg), München 2020, S. 559f. (Nr. 8I.0.3) und Abb. I64.

4 <https://www.sub.uni-goettingen.de/projekte-forschung/projektdetails/projekt/katalogisierung-der-abendlaendischen-mittelalterlichen-handschriften-der-niedersaechsischen-staats/> (28.8.202I).

5 Vgl. Mone [Anm. 3], Sp. 3II.

6 So schon Meyer [Anm. 3], S. 545, und Schorbach [Anm. I], S. 269, Anm. 2.

7 Helmut Kind und Jochen Bornmüller, Incunabula Gottingensia. Inkunabelkatalog der Niedersächsischen Staatsund Universitätsbibliothek Göttingen, Bd. 2: Abteilung Critica bis Jus, Wiesbaden 2006, S. $278 f$.

8 Vgl. Schorbach [Anm. I], S. 269, Anm. 2.

9 Vgl. Ulmschneider [Anm. I], S. II3. 
Io Vgl. Ernst Kyriss, Deutsche Buchbinder der Spätgotik und Renaissance, in: Archiv für Geschichte des Buchwesens 3 (196I), Sp. 18I-204, hier Sp. I84, Nr. I8: „Esch Jodocus, vielleicht Basel: Heidelberg UB Inc. Q 300 ${ }^{1 / 11} 2$ Bände um I475“.

II Vgl. Kyriss [Anm. Io].

I2 Vgl. Armin Schlechter und Ludwig Ries, Universitätsbibliothek Heidelberg. Katalog der Inkunabeln der Universitätsbibliothek Heidelberg, des Instituts für Geschichte der Medizin und des Stadtarchivs Heidelberg, Teil I (Inkunabeln in Baden-Württemberg. Bestandskataloge 3,I), Wiesbaden 2009, S. 258, Nr. 279.

I3 Dazu wird in Kürze ein ausführlicher Beitrag an anderer Stelle erscheinen.

14 Schorbach [Anm. I], S. 27 If. 